\title{
Comparative Analysis on Mitochondrial Control Region DNA Diversity of Three Far Eastern Catfish (Silurus asotus) Populations
}

\author{
Zhuang Xue ${ }^{1}$, Yuying Zhang ${ }^{2}$, Haikun Sun ${ }^{1}$, Wei Wang ${ }^{1,2, *}$ (i) \\ ${ }^{1}$ Dalian Ocean University, College of Fisheries and Life Science, Dalian 116023, China \\ ${ }^{2}$ Florida International University, Department of Biological Sciences, North Miami, 33181, USA
}

\section{Article History}

Received 15 April 2019

Accepted 07 January 2020

First Online 17 January 2020

\section{Corresponding Author}

Tel.: +8641184762236

E-mail: wangwei@dlou.edu.cn

\section{Keywords}

Silurus asotus

D-loop

Genetic diversity

Genetic structure

Population expansion

\begin{abstract}
To investigate genetic diversity of the far eastern catfish (Silurus asotus) populations in the Yellow River, the Songhua River, and the Dayanghe River, mitochondrial control region (D-loop) DNA was analyzed for the samples collected in Zhengzhou (ZZ in the Yellow River), Haerbin (HEB in the Songhua River), and Donggang (DG in the Dayanghe River). There were totally twenty-one haplotypes identified in the three groups of samples. Haplotype networks by using neighbor-joining and median-joining clustering methods both indicated that the haplotypes 3,8 , and 19 constituted a single branch and the other 18 haplotypes constituted another branch. The highest genetic differentiation coefficients were observed between the $Z Z$ and HEB samples (0.264), and the lowest was between the $Z Z$ and $D G(0.101)$. The average gene flow among the three populations was 3.063. The Fu's Fs and Tajima's D test values of the three populations were both negative, and indicated no significant difference between three populations ( $p=0.08$ and $0.06>0.05$ ). Our results suggest that the far eastern catfish populations have not undergone significant population expansion and remained stable in the Yellow River, the Songhua River, and the Dayanghe River.
\end{abstract}

\section{Introduction}

The catfish (Silurus asotus), a member of the family Siluridae, is a freshwater species that is abundant and widely distributed throughout China, the Korean Peninsula, the Japanese islands, and the eastern regions of Russia. In the past several decades, catfish populations have been threatened by overfishing, water pollution, and water conservation projects ( $\mathrm{Hu}, \mathrm{Pan}$, Hou, \& Li, 2002; Yan, Jiang, \& Liu, 2004). It is important to better understand the population dynamics of $S$. asotus, especially regarding the status of genetic diversity, and the conservation of endemic germplasm resources, with the purposes of sustaining the catfish populations.

To survey the extant natural resources of $S$. asotus is challenging, which requires acquisition of large amounts of data of genetic information from various catfish populations. The RAPD marker (Yoon, \& Kim 2001), cytochrome B sequences of mitochondrial DNA (Xiao, Cui, Kang \& Zhang, 2013; Xu, 2013), and microsatellite markers ( $\mathrm{Wu}, \mathrm{Lian}, \mathrm{Hou}, \mathrm{Li}, \&$ Xiao, 2011) have previously been used to study the genetic diversity of catfish and related species. These methods have provided a wealth of information regarding the genetic fitness of these species, but the analysis of mitochondrial control region (D-Loop) DNA in catfish has not been reported. The D-loop region of mitochondrial DNA has been widely used in population genetic analysis, because it accumulates mutations more rapidly than other genomic regions and is not affected by selective pressure, as the $D$-loop region does not code for any protein (Xiao, Zhang, \& Gao, 2009; Yuan, Zhang, Li, Zhu, \& Luo, 2010). 
The Songhua River, the Yellow River, and the Dayanghe River are chief rivers in the northern China, and contain rich catfish resources. In this study, the mitochondrial D-Loop was used to analyze the population genetic diversity and adaptive variation of three catfish populations in Zhengzhou (ZZ) of the Yellow River, Haerbin (HEB) of the Songhua River, and Donggang (DG) of the Dayanghe River. The results will be useful to inform catfish germplasm resource management, and better conserve the biodiversity and fitness of catfish populations.

\section{Materials and Methods}

\section{Animals}

The $S$. asotus samples were obtained from three catfish populations in ZZ, HEB and DG in 2017 (Figure 1).
Thirty 1-2 age adults were selected from each of the population. The average body length is $254.83 \pm 41.07$ $\mathrm{mm}$, and the body weight is $158.73 \pm 90.86 \mathrm{~g}$. The backmuscle tissues were collected from 15 catfishes in each of the populations and stored in $95 \%$ ethanol prior to DNA extraction. All surgeries were performed under sodium pentobarbital anesthesia, and every effort was taken to minimize suffering.

\section{Extraction of Genomic DNA}

Total genomic DNA was extracted from the backmuscle samples using the Animals DNA extraction kit (Tiangen biotech(Beijing)Co., LTD). Total DNA was adjusted to a concentration of $50 \mathrm{ng} / \mathrm{ml}$ and stored in $\mathrm{ddH}_{2} \mathrm{O}$ (Mascolo et al., 2019). The DNA quantity was measured by nanodrop 2000 and agarose gel electrophoresis (Figure 2).

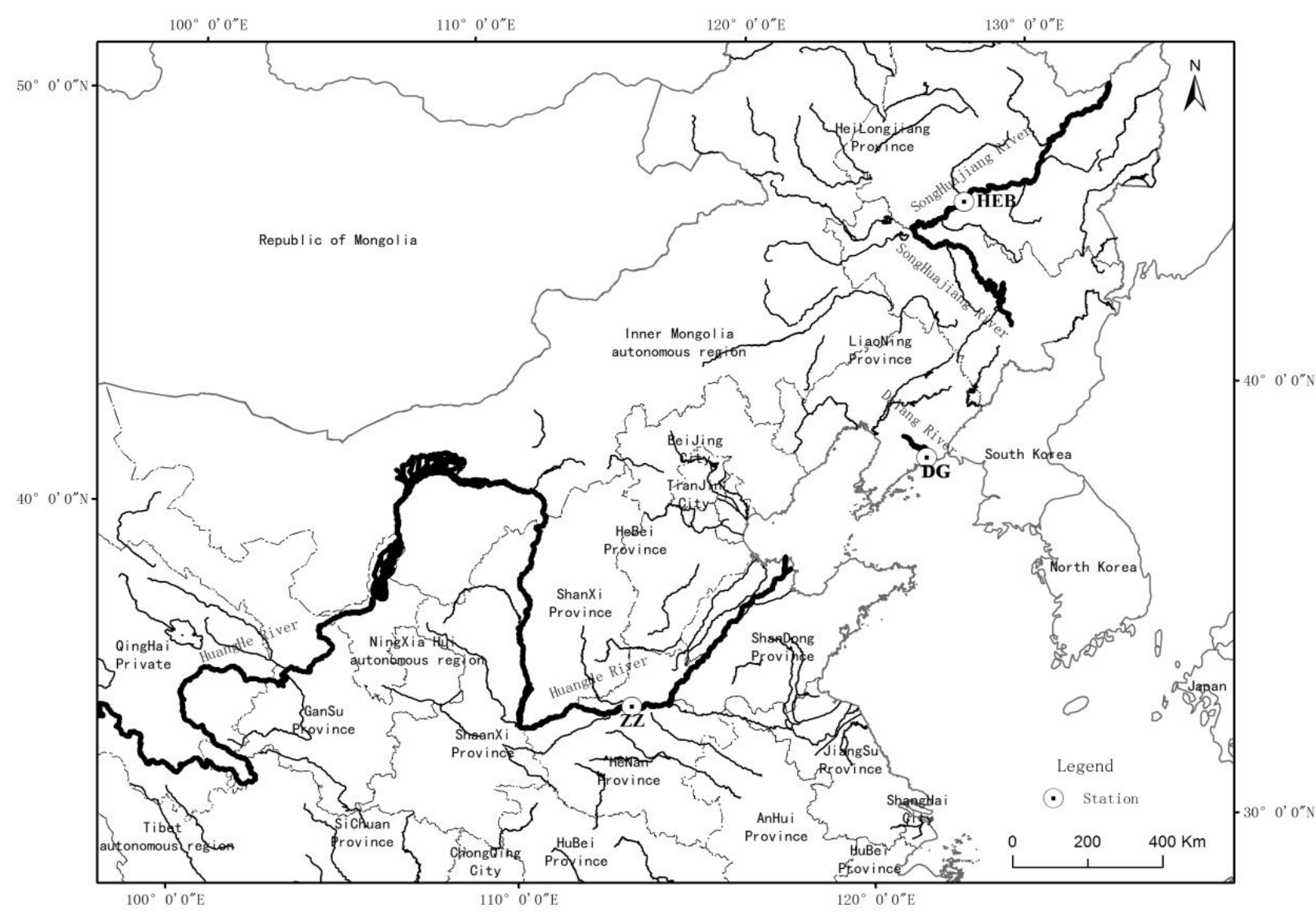

Figure 1. Sampling locations of the 3 Silurus asotus populations.

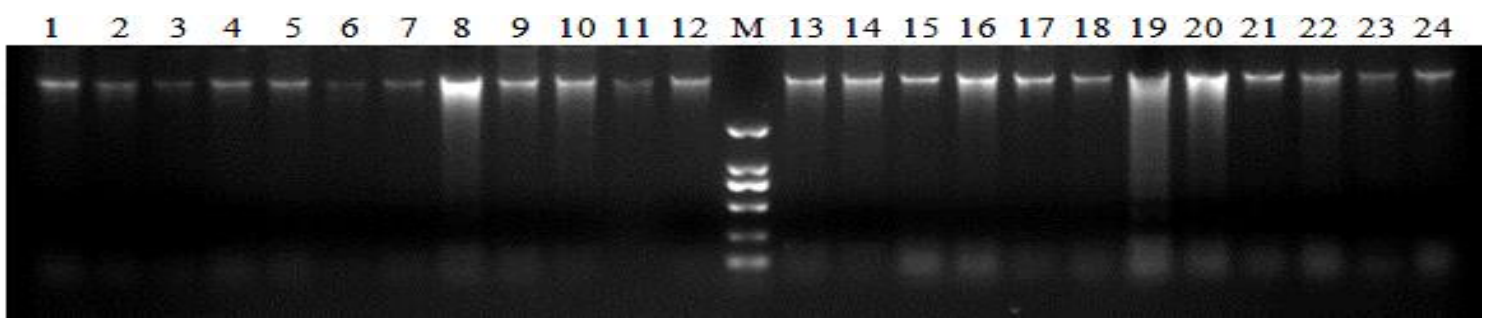

Figure.2. The electrophoretic pattern of Silurus asotus genomic DNA (portion). Back muscle tissue genomic DNA of Silurus asotus was extracted and electrophoresed on a $1.8 \%$ agarose gel. M: Marker (2000, 1000, 750, 500, 300, 100 bp). 


\section{Amplification of D-Loop Sequences}

The mitochondrial D-loop was amplified by polymerase chainreaction (PCR) using the following primer set: forward primer 5'AGGGGTATGTGGAGTAAA-3' and reverse primer 5'AGgGGTATGTGGAGTAAA-3'. The primers were designed by the software of Primer 5.0 (Canada, Premier 5.0). Amplification was carried out in $50 \mu \mathrm{L}$ reaction volume containing the following components: $1 \mu \mathrm{l}$ genomic DNA, $25 \mu$ l Easy Taq ${ }^{\circledR}$ PCR Super Mix (Easy Taq ${ }^{\circledR}$ PCR System; TaKaRa, Dalian, China), $1.5 \mu \mathrm{l}$ of each

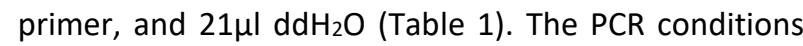
were set asfollows: $94^{\circ} \mathrm{C}$ for 2 min followed by 33 cycles of denaturing at $94^{\circ} \mathrm{C}$ for $45 \mathrm{~s}$, annealing at $56^{\circ} \mathrm{C}$ for $1 \mathrm{~min}$, and elongating at $72^{\circ} \mathrm{C}$ for $1 \mathrm{~min}$, then a final extension at $72^{\circ} \mathrm{C}$ for $8 \mathrm{~min}$. Amplified products were purified with a Gel Extraction Kit (Generay Biotech Co., Ltd. Shanghai). Then, the PCR products were sent to the Generay Biotech Co. Ltd. for sequencing.

\section{Sequence Alignments and Analyses}

The sequences were proofread, edited, and aligned using BioEdit software version 7.0.5.3 (Ibis Biosciences, Carlsbad, CA). Mismatch distribution analysis of these polymorphisms was assessed using DnaSP v 5.0 (Rozas, Sánchez-Delbarrio, \& Messeguer, 2003) (Univers it at de Barcelona, Barcelona, Spain). Historical population expansion was examined using Tajima's and Fu's statistics with 10,000 demographic history records among the three populations. Parameters of genetic diversity within and between populations, such as the haplotype diversity (HD), nucleotide diversity $(\pi)$, average number of nucleotide difference $(\mathrm{K})$, and gene flow $(\mathrm{Nm})$, were estimated with MEGA (Tamura, Peterson, \& Peterson, 2011) (version 5.05). Phylogenetic reconstruction of the haplotypes was conducted using the maximum likelihood method, and a consensus of 10,000 bootstrap replicates. Arlequin (version 3.11; University of Berne, Berne, Switzerland) was used to estimate the genetic differentiation and Fst values with an analysis of molecular variance (Excoffier, Laval, \& Schneider, 2005) (AMOVA). A haplotype network of the three catfish populations was constructed by Network (Bandelt, Forster, \& Röhl, 1999). Statistical analyses were performed using SPSS19.0.

\section{Results}

\section{Composition Analysis of the D-Loop Sequence}

The D-Loop position for catfish between tRNA Pro and tRNA Phe. Approximately 535bp of the mtDNA control region, which shares extensive homology with other catfish populations (Mascolo et al., 2018), was analyzed from the ZZ (12), HEB (14), and DG (15) catfish populations. A total of 41 effective sequences were obtained and analyzed, and the estimated transition/transversion bias $(R)$ was 6.13; $\mathrm{A}=29.6$, $\mathrm{C}=27.8, \mathrm{G}=14.9$, and $\mathrm{T}=27.8 ; \mathrm{A}+\mathrm{T} \quad(64.8 \%)$ was significantly higher than $C+G(35.2 \%)$ (Table 2).

\section{Analysis of Genetic Diversity and Population Genetics Structure}

The estimates of haplotype diversity and nucleotide diversity are shown in Table 3. The DG population showed the highest HD $(0.924 \pm 0.053)$, the

Table 1. Reaction system and primer sequences of Silurus asotus D-loop PCR

\begin{tabular}{lc}
\hline Component & Volume \\
\hline Genomic DNA(50ng/ $\mu$ l) & $1 \mu \mathrm{l}$ \\
F-Primer(5' -AGGGGTATGTGGAGTAAA-3' ) & $1.5 \mu \mathrm{l}$ \\
R-Primer(5' -AGGGGTATGTGGAGTAAA-3' ) & $1.5 \mu \mathrm{l}$ \\
$2 \times 5^{\prime}$ EasyTaq ${ }^{\prime}$ PCRSuperMix & $25 \mu \mathrm{l}$ \\
$\mathrm{ddH}_{2} \mathrm{O}$ & $21 \mu \mathrm{l}$ \\
\hline
\end{tabular}

Table 2. Nucleotide composition of the D-LOOP sequences from three Silurus asotus groups (\%)

\begin{tabular}{lccccc}
\hline Population & T & C & A & G & G+C \\
\hline ZZ & 29.8 & 20.4 & 35.0 & 35.2 & 34.8 \\
DG & 29.9 & 20.4 & 34.9 & 14.8 & 35.2 \\
HEB & 29.7 & 20.5 & 35.0 & 35.3 & 14.8 \\
\hline
\end{tabular}

Table 3. Genetic diversity of the D-LOOP sequences from three Silurus asotus populations

\begin{tabular}{|c|c|c|c|c|}
\hline Population & Haplotype $(H)$ & $\begin{array}{l}\text { Haplotype diversity } \\
\qquad(H D)\end{array}$ & Polymorphic digit $(P)$ & Nucleotide diversity $(\pi)$ \\
\hline $\mathrm{ZZ}$ & 3 & $0.591 \pm 0.108$ & 11 & $0.007 \pm 0.002$ \\
\hline DG & 10 & $0.924 \pm 0.053$ & 28 & $0.013 \pm 0.002$ \\
\hline HEB & 8 & $0.890 \pm 0.060$ & 19 & $0.012 \pm 0.001$ \\
\hline
\end{tabular}


highest polymorphic digit $(P=28)$, and the highest nucleotide diversity $(\pi=0.013 \pm 0.002)$. The genetic diversity of the three catfish populations was ranked as: DG, HEB, and ZZ.

The analysis resulted in 21 haplotypes from 41 individuals of S. asotus. Haplotype Hap_20 was the most frequent one, and the distribution of haplotypes was unique within each population. No same haplotype was shared among all three catfish populations. Ten haplotypes were observed in the DG population, the most in any of the populations, and only three haplotypes were observed in the ZZ population, which is the least in any of the catfish populations (Table 4).

The genetic distance of the three populations was 0.011-0.015; the largest genetic distance was between the HEB and ZZ populations, and the smallest genetic distance was between the $Z Z$ and DG populations (Table 5).

The UPGMA tree was constructed based on the genetic distance of the three populations of catfish, and showed that all the three populations were assigned to two clades, associated with a dispersion and expansion of the population, indicating the close relationship between the DG and ZZ populations (Figure 3).

Twenty-one kinds of haplotypes were found in three catfish populations, Hap_3 and Hap_8 were only found in DG population; Hap_19 is a haplotype unique to the ZZ population. The number of clustered haplotypes accounted for $86 \%$ of the total haplotypes. Cluster analysis of the haplotypes by neighbor-joining using the bootstrap method (repeat number 10000) test and it is shown in Figure 4.

Table 4. Haplotype distribution in three Silurus asotus populations

\begin{tabular}{lccc}
\hline Haplotype & & Haplotype distribution in population & HEB \\
\cline { 2 - 4 } & ZZ & 4 & 0 \\
\hline Hap_1 & 0 & 1 & 0 \\
Hap_2 & 0 & 2 & 0 \\
Hap_3 & 0 & 1 & 0 \\
Hap_4 & 0 & 1 & 0 \\
Hap_5 & 0 & 1 & 0 \\
Hap_6 & 0 & 1 & 0 \\
Hap_7 & 0 & 1 & 0 \\
Hap_8 & 0 & 1 & 0 \\
Hap_9 & 0 & 2 & 0 \\
Hap_10 & 0 & 0 & 1 \\
Hap_11 & 0 & 0 & 3 \\
Hap_12 & 0 & 0 & 2 \\
Hap_13 & 0 & 0 & 1 \\
Hap_14 & 0 & 0 & 1 \\
Hap_15 & 0 & 0 & 1 \\
Hap_16 & 0 & 4 \\
Hap_17 & 0 & 1 \\
Hap_18 & 0 & 0 \\
Hap_19 & 0 & 0 \\
Hap_20 & 0 & 0 \\
Hap_21 & 4 & 0 & 0 \\
\hline
\end{tabular}

Table 5. Pairwise K2-P genetic distances (lower left) and Fst (upper right) between three Silurus asotus populations

\begin{tabular}{cccc}
\hline Population & ZZ & DG & HEB \\
\hline ZZ & - & 0.101 & 0.264 \\
DG & 0.011 & - & 0.130 \\
HEB & 0.015 & 0.013 & - \\
\hline
\end{tabular}

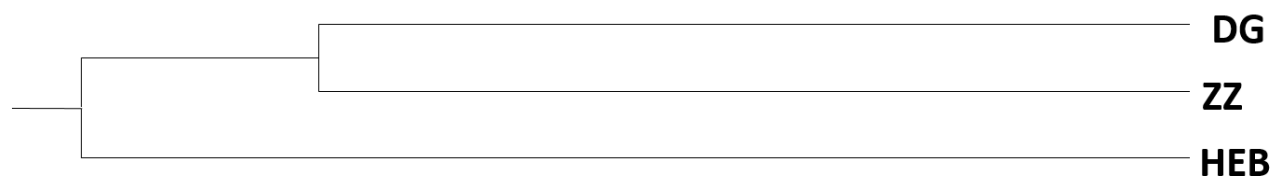

\begin{tabular}{lllllll}
$\mid$ & $\mid$ & & & & & $\mid$ \\
\hline .006 & 0.005 & 0.004 & 0.003 & 0.002 & 0.001 & 0.000
\end{tabular}

Figure.3. The UPGMA clustering tree of three Silurus asotus populations based genetic distance 
Network analysis was used to identify the phylogenetic relationships among the 21 haplotypes within the three populations. The network analysis indicates that all haplotypes were divided into two groups, haplotype 3, 8 and 19 belongs to group A, and the other haplotypes belongs to group $B$ (Figure 5 ). This is consistent with the phylogenetic analysis (Figure 4).
The distribution frequency of haplotype 19 was $57 \%$ of the total A group, and haplotype 19 was the dominant haplotype. The distribution frequency of haplotype 1 , 17 , and 20 were $4 \%$ of the total $B$ group; these three made up the dominant haplotypes. The dominant haplotypes within the DG, HEB, and ZZ populations were Hap_1, Hap_17, and Hap_20, respectively.

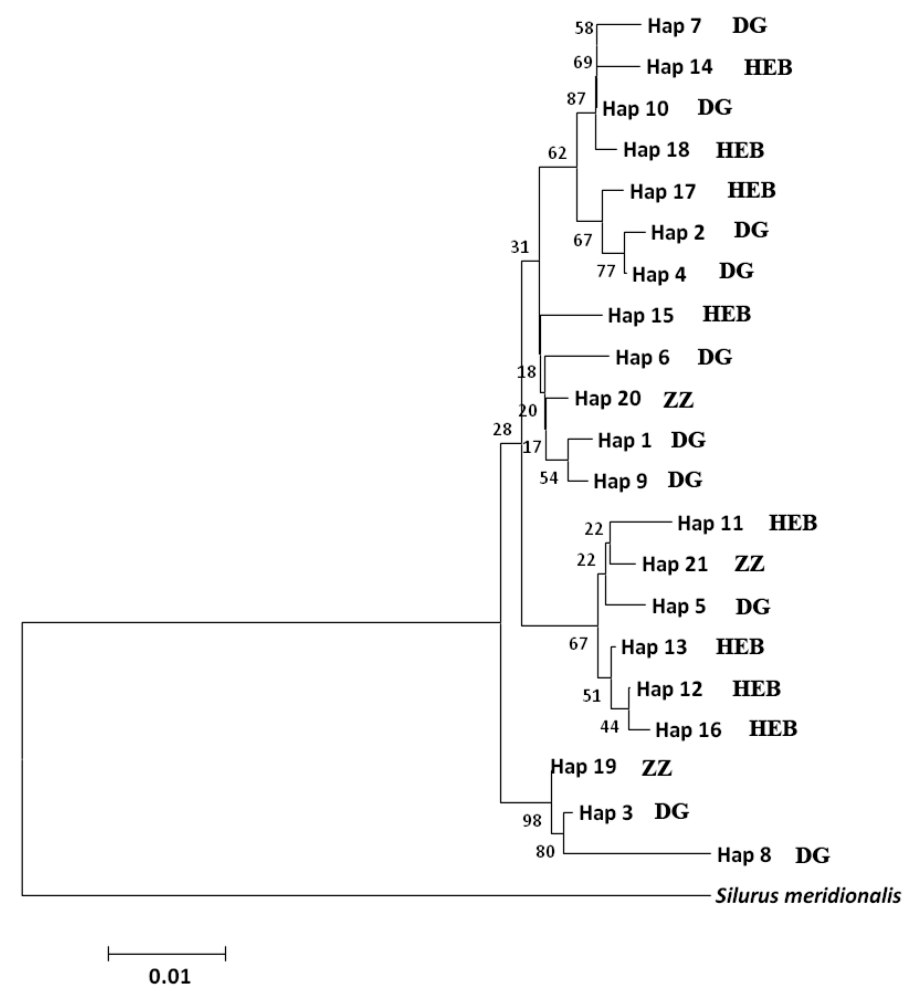

Figure.4. NJ phylogeny tree of D-loop haplotype of three Silurus asotus populations

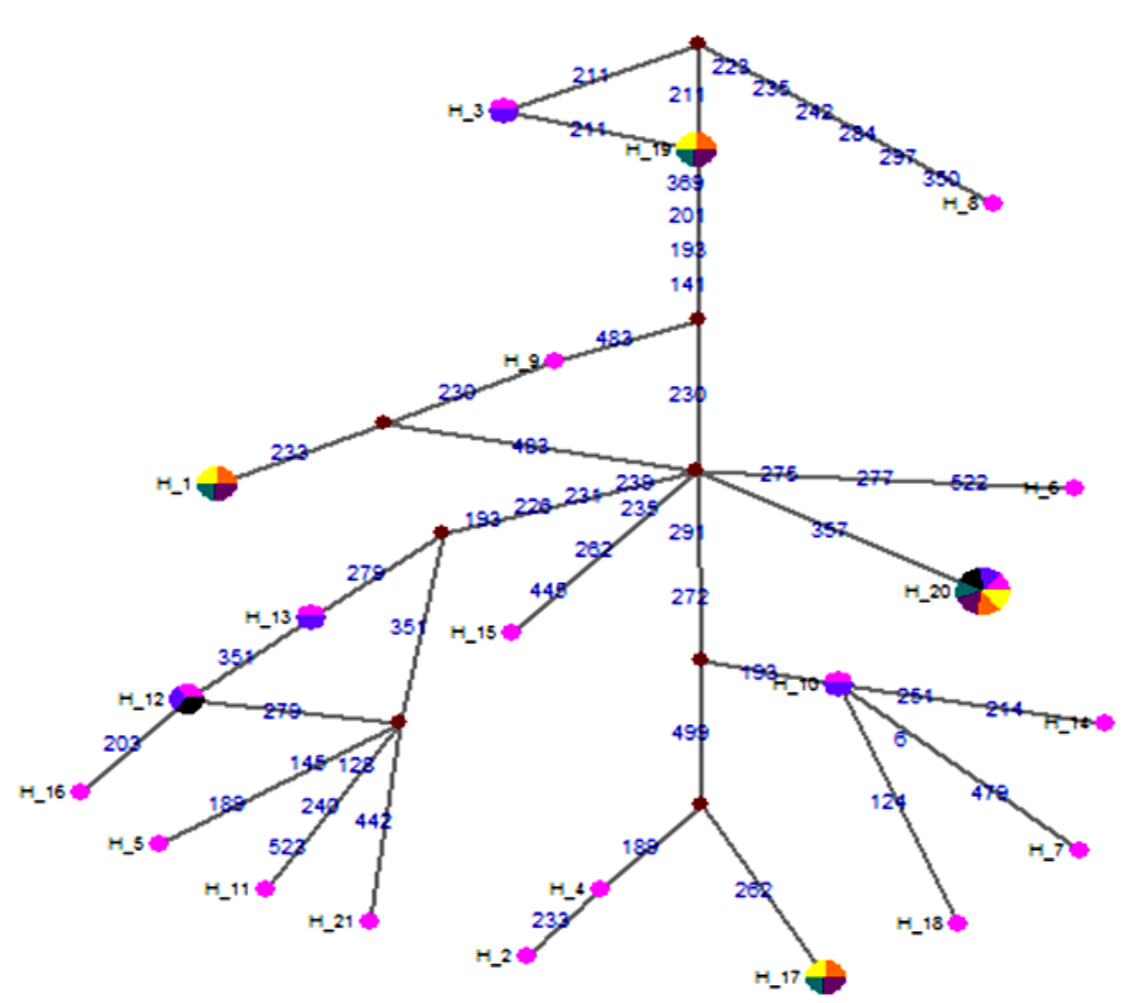

Figure.5. Median joining network of three Silurus asotus populations 


\section{Analysis of Molecular Variance}

The significance of the three population structures based on haplotype frequency: $16.32 \%$ molecular variance was attributed to the differentiation between the populations, whereas $83.68 \%$ molecular variance was observed within the populations (Table 6). Population structuring revealed that the Fst value was not significant $(p=0.09>0.05)$ (Table 5$)$. The genetic differentiation coefficient of the three populations ranged from $0.101-0.264$, and the average value was 0.165 . The genetic differentiation coefficient between the $Z Z$ and HEB populations was the highest ( $F s t=0.264$ ), and was lowest between the DG and ZZ populations (Fst=0.101).

The tests of neutral evolution (Tajima's D and Fu's $F$ tests) indicated that there was no significant difference $(p=0.08$ and 0.06$)$ among the three populations, indicating no significant population expansion. Mismatch distribution analysis of the mitochondrial D-loop regions sequences revealed no peaks (Figure 6).

\section{Discussion}

Genetic diversity of three $S$. asotus populations was assessed by analyzing the mtDNA D-loop sequences of 41 individuals from three populations. Within these three catfish populations the $\mathrm{G}+\mathrm{C}$ content in the mtDNA $D$-loop region was significantly lower than the $A+T$ content, which was consistent with the results of base composition of mitochondrial cytochrome B in a study of catfish from the Huaihe River (Xiao et al., 2013). The $R$ of the three populations was larger than 2.0, indicating that the mitochondrial D-loop sequence can be used to assess genetic diversity between these populations (Kumar, Tamura, \& Nei, 2004).

The haplotype diversity (HD) and nucleotide diversity in the DG and HEB populations was similar to previously reported (Xu et al., 2013), which identified haplotype diversity and nucleotide diversity of cyochrome $B$ sequences from multiple Chinese catfish populations ( $H D=0.948 \pm 0.009, \pi=0.018 \pm 0.001)$. The HD and $\pi$ described for the catfish populations in this study are higher than those reported in carp populations from the Yangtze and Pearl rivers (Fu, 2011). The genetic diversity of a species is closely related to the ability of that species to adaptation and survive to changing environmental conditions (Conrad, 1983; Féral, 2002; Frankham, Ballou, \& Briscoe, 2002), and low diversity can indicate a decline in how well a species can adapt to environmental change (Nei, 1978; Gu, 2004). However, the values of $H D$ and $\pi$ of $Z Z$ the population were significantly lower than those of the DG and HEB populations, indicating that the DG and HEB catfish populations may be better equipped to adapt to environmental changes than the $\mathrm{ZZ}$ population, due to higher genetic diversity within those populations. The low genetic diversity of the $Z Z$ population may be related to environmental pollution and ecological damage in the Yellow River, and may also be caused by environmental deterioration of the Yellow River (Guan et al., 2016; Michel, Wolters, Sun, Huang, \& Kuenzer, 2016).

The study of the genetic structure of a species can provide critical information regarding the genetic diversity within an individual population and between geographically diverse populations (Yan, Song, Wang, Lu, \& Gao, 2015). Within these three catfish populations in the present study, the average individual genetic distance was 0.011 , indicative of small differences between populations. In general, the genetic distance values of a genus, species, and population are 0.90, 0.30 and 0.05(Shaklee, Tamaru, \& Waples, 1982), respectively; the genetic distance between the three catfish populations was less than 0.05. Although the HEB and DG catfish populations are nearest in geographic proximity, the genetic distance between these populations was the greatest, indicating that the size of genetic distance between catfish populations does not necessarily correspond to the geographic distance between those populations. However, although analysis of molecular variation revealed that most variation arises from within an individual catfish population, and the overall variation between populations was small. Ultimately, no significant genetic differentiation was identified among the three catfish populations studied.

The value of gene flow is related to the exchange of genetic information between populations. Populations are isolated when the $\mathrm{Nm}$ is less than 1 ; an $\mathrm{Nm}$ greater than 1 indicates gene flow between populations; and an $\mathrm{Nm}$ greater than 4 indicates that each population exists as a random unit (Masatoshi, \& Sudhir, 2000). In the current study, the $\mathrm{Nm}$ between $\mathrm{ZZ}$ and HEB was the smallest, but still greater than 1, indicating evidence of some gene flow between the two populations. The $\mathrm{Nm}$ between $\mathrm{ZZ}$ and DG was more than 4 , which showed that two populations form a random unit. The gene flow between the three populations is closely related to the genetic differentiation of the

Table 6. The analysis of molecular variance from three Silurus asotus populations

\begin{tabular}{lcccc}
\hline Source of Variation & $d f$ & Sum of squares & Variance components & Percentage of variation,(\%) \\
\hline Populations & 2 & 23.634 & 0.631 & 16.32 \\
Within populations & 38 & 122.879 & 3.234 & 83.68 \\
Total & 40 & 146.512 & 3.864 & \\
\hline
\end{tabular}



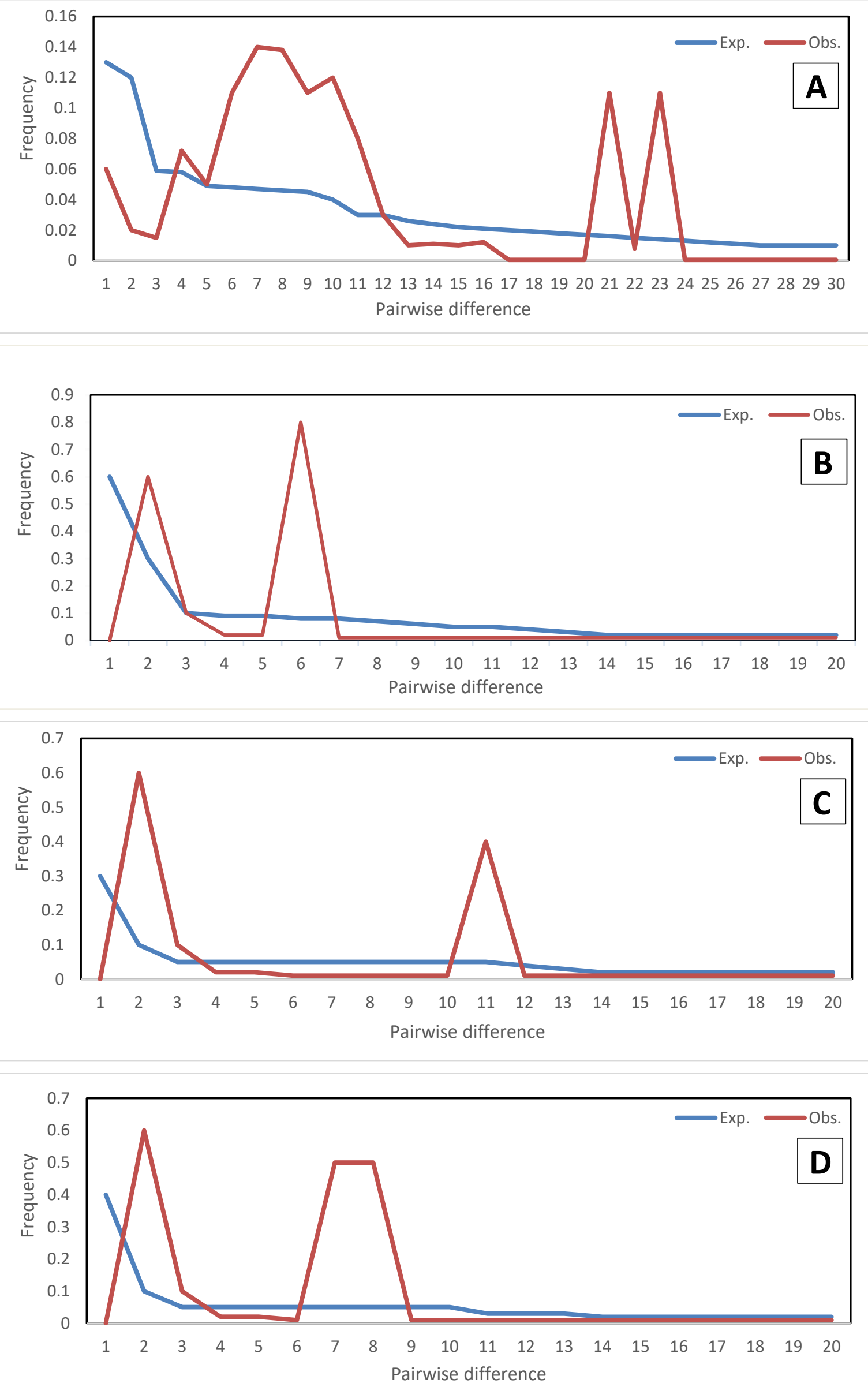

Figure 6. The mismatch distribution of D-LOOP sequence of three Silurus asotus populations Note: A. Total, B. HEB, C. DG, D. ZZ. 
populations, and may be the reason for the small genetic differentiation among the three catfish populations. In addition, the gene flows among the three populations do not correspond with geographical distance; factors such as commodity circulation, enhancement, and artificial breeding of catfish may cause this phenomenon.

The network method is commonly used to analyze molecular variation analysis of the species, and is especially suitable for the analysis of non-recombinant DNA sequences, such as mitochondrial DNA and $Y$ chromosomal DNA (Bandelt et al., 1999). Phylogenetic relationships of 21 haplotypes were analyzed by adjacent tree and intermediary network diagramming in the present study. However, the intermediary network figure reveals that haplotype did not correspond to sampling sites. Hap_20, Hap_19, Hap_17, and Hap_1 have the highest frequency, suggesting these may be the original haplotypes.

The tests of neutral evolution (Tajima's D and Fu's Fs) were both negative in our study, which is consistent with Liu' report (Liu, Yang, Wang, \& Tang, 2009). Distribution of pairwise mismatches of $S$. asotus populations was multimodal, indicating no significant population expansion in the three rivers. Thus, it was concluded that the genetic differentiation among the three catfish populations was low.

The behavior of genetic variation within S. asotus populations provides important information in developing the conservation and management strategies for endangered fish populations. Despite the identification of the low levels of genetic differentiation among the $Z Z, H E B$, and DG catfish populations, the results indicate that geographic and environmental features of drainage regions may significantly impact the different catfish populations. Furthermore, these results suggest that conservation stations should be established on the three rivers to increase the number of catfish populations, and to improve the genetic diversity and overall fitness of this species. The results also improved the understanding about role of genetic variation in the catfish conservation. Genetic variation is a dimension of the biodiversity, to better monitor and compare the biodiversity of $S$. asotus populations the mechanism that driving the genetic variation should be researched in the future.

\section{Acknowledgments}

We would like to express our sincere gratitude to the editor and reviewers who have spent considerable time and effort in this paper. We are also grateful to the professional editing service (Elsevier Language Editing Services) for improving the language of our manuscript. This work was supported by the National Natural Science Foundation of China (41101481), and the National High-Tech R\&D Program (863 Program; 2012AA10A413-3).

\section{References}

Bandelt, H.J., Forster, P., \& Röhl, A. (1999). Median-joining networks for inferring intraspecific phylogenies. Molecular Biology \& Evolution, 16, 37-48. https://doi.org/10.1093/oxfordjournals.molbev.a026036

Mascolo, C., Ceruso, M., Chirollo, C., Palma, G., Anastasio, A., Pepe, T., Sordino, P. (2018). The complete mitochondrial genome of the Angolan dentex Dentex angolensis (Perciformes: Sparidae). Journal Mitochondrial DNA Part B: Resources, 3, 391-392. https://doi.org/10.1080/23802359.2018.1450675

Mascolo, C., Ceruso, M., Sordino, P., Palma, G., Anastasio, A., Pepe, T. (2019). Comparison of mitochondrial DNA enrichment and sequencing methods from fish tissue. Food Chemidtry, 294, 333-338. https://doi.org/10.1016/j.foodchem.2019.05.026

Conrad, M. (1983). Adaptability: the Significance of Variability from Molecular to Ecosystem. Bulletin of Mathematical Biology, 48, 701-703. https://doi.org/10.1007/BF02462331.

Excoffier, L., Laval, G., \& Schneider, S. (2005). Arlequin ver. 3.0: An integrated software package for population genetics data analysis. Evolutionary Bioinformatics Online, 1:4750. https://doi.org/10.1177/117693430500100003.

Féral, J.P. (2002). How useful are the genetic markers in attempts to understand and manage marine biodiversity? Journal of Experimental Marine Biology \& Ecology, 268: 121-145. https://doi.org/10.1016/s00220981(01)00382-3.

Frankham, R., Ballou, J.D., \& Briscoe, D.A. (2002). Introduction to conservation genetics. Cambridge, Cambridge University Press, $641 \mathrm{pp}$.

Fu, X.Y. (2011). Genetic diversity of mitochondrial cytochromes $b$ in black carp from the Yangtze and the Pearl River (Master's thesis). Jinan University, Jinan, China.

Guan, Q.Y., Wang, L., Pan, B.T., Guan, W.Q., Sun, X.Z., \& Cai, A. (2016). Distribution features and controls of heavy metals in surfaces ediments from the river bed of the Ningxia-Inner Mongolian reaches, Yellow River, China. Chemosphere, 144: 29-42.

https://doi.org/10.1016/j.chemosphere.2015.08.036.

Gu, W.C. (2004). Satistical Genetics. Beijing, China, Science Press., $422 \mathrm{pp}$.

Hu, X.J., Pan, T.S., Hou, G.J., \& Li, H.Y. (2002). The status and protection of wild catfish resource in Anhui province. Journal of Anhui Agricultural Sciences, 30: 233-247. https://doi.org/10.3969/j.issn.0517-6611.2002.02.034.

Kumar, S., Tamura, K., \& Nei, M. (2004). MEGA3: Integrated software for molecular evolutionary genetics analysis and sequence alignment. Briefings in Bioinformatics, 5:150-163. https://doi.org/10.1093/bib/5.2.150.

Liu, Z.Z., Yang, J.Q., Wang, Z.Q., \& Tang, W.Q. (2009). Genetic Structure and Population History of Beleophthalmus petinirostris in Yangtze River Estuary and Its Southern Adjacent Regions. Zoological Research, 30: 1-10. https://doi.org/10.1016/S1874-8651(10)60059-2.

Masatoshi, N., \& Sudhir, K. (2000). Molecular Evolution and Phylogenetics. Oxford, England, Oxford University Press, $174 \mathrm{pp}$.

Michel, L., Wolters, Sun, Z.C., Huang, C., \& Kuenzer, C. (2016). Environmental awareness and vulnerability in the Yellow River Delta: Results based on a comprehensive 
household survey. Ocean Coastal Manage, 120: 1-10. https://doi.org/10.1016/j.ocecoaman.2015.11.009.

Nei, M. (1978). Estimation of average heterozygosity and genetic distance from a small number of individuals. Genetics, 89: 583-590.

https://doi.org/10.0000/PMID17248844.

Rozas, J., Sánchez-Delbarrio, J.C., \& Messeguer, X. (2003). DnaSP, DNA polymorphism analyses by the coalescent and other methods. Bioinformatics, 19: 2496-2497. https://doi.org/10.1079/9780851994758.0139.

Shaklee, J.B., Tamaru, C.S., \& Waples, R.S. (1982). Speciation and Evolution of Marine Fishes Studied by the Electrophoretic Analysis of Proteins. Pacificence, 36: 141-157.

https://doi.org/10.1016/0077-7579(82)90068-0.

Tamura, K., Peterson, D., \& Peterson, N. (2011). MEGA5: Molecular evolutionary genetics analysis using maximum like lihood, evolutionary distance, and maximum parsi-mony methods. Molecular Biology \& Evolution, 28: 2731-2739. https://doi.org/10.1093/molbev/msr121.

Wu, X.D., Lian, Z.Q., Hou, Y.X., Li, L., \& Xiao, W. (2011). Application analysis of microsatellite markers from Silurus meriaionalis on three species of Siluriformes. Acta Hydrobiologica Sinica, 35: 638-645. https://doi.org/10.1631/jzus.B1000171.

Xiao, M.S., Cui, F., Kang, J., \& Zhang, X.H. (2013). Genetic Structure and Variation of Wild Chinese Catfish from Huaihe River Based on Cytochrome b Gene Sequences. Chinese Journal of Zoology, 48: 75-86. https://doi.org/10.13859/j.cjz.2013.01.016
Xiao, Y.S., Zhang, Y., \& Gao, T.X. (2009). Genetic diversity in the mtDNA control region and population structure in the small yellow croaker Larimichthys polyactis. Environmental Biology of Fishes, 85: 303-314. https://doi.org/10.1007/s10641-009-9497-0.

Xu, D. (2013). Genetic diversity of Amur catfish (Silurus asotus) based on microsatellite markers and cytochrome $b$ sequences (Master's Thesis). Southwest University, Chongqing, China.

Yan, L., Song, N., Wang, J., Lu, Z.C., \& Gao, T.X. (2015). Studies on genetic diversity of Chinese mitten crab Eriocheir sinensis based on mitochondrial DNA control region. Acta Hydrobiologica Sinica, 39: 615-620. https://doi.org/10.7541/2015.81.

Yan, W.J., Jiang, Y.G., \& Liu, D. (2004). Water environment pollution and control counter measures in Huaihe River near Anhui Province. Journal of Soil Water Conservation, 18: 21-24.

https://doi.org/10.13870/j .cnki .stbcxb.2004.02.006

Yoon, J.M., \& Kim, G.W. (2001). Randomly amplified polymorphic DNA-polymerase chain reaction analysis of two different populations of cultured Korean catfish Silurus asotus. Journal of Biosciences, 26: 641-647. https://doi.org/10.1007\%2FBF02704762.

Yuan, J., Zhang, Q.Z., Li, F., Zhu, C.K., \& Luo, F. (2010). MtDNA control region sequence variation and genetic diversity of Coreius Heterodon (Bleeker) in the upper and middle sections of the Yangtze River. Acta Hydrobiologica Sinica, 34: 9-19.

https://doi.org/10.3724/SP.J.1035.2010.00009. 